\title{
Health Coaching in Psychiatry: Personal Experience and Literature Reviews.
}

\author{
Lamichhane $\mathbf{N}$
}

Associate Professor, Dept. Of Psychiatry, Gandaki Medical College, Pokhara, Nepal

E-mail *Corresponding author: drnrmlam@hotmail.com

\section{INTRODUCTION:}

Recovery focused services are a central component to making the mental health services fit for the twenty-first century. Whilst the concept of Recovery is not new, as psychiatrists we need to rethink how we work alongside, in partnership with, people who use our services to enable them to get on life from the point when they first developed the condition i.e. to attain their premorbid level of functioning in the community. When health coaching services are delivered

to individuals with one or more chronic conditions , professionals are typically practicing preventati ve and therapeutic health coaching 2 . According to Hudson, 1999 and Tobias, 1996 although the origins of health coaching began back in the 1940s, it wasn't until the 1980s that the field really came into its own stature 4 . Skillful conversation and clinical interventions used to actively and safely engage patients in selfmanagement of health conditions and health behavior changes, with the goal to improve health outcomes, lower health risk and reduce overall costs. The health coach in psychiatry will primarily focus on providing high quality, cost effective mental health services to individuals by working with clients to develop personal, professional and mental health goals and developing treatment plans. The coach for this position should have mental health services experience which can be leveraged to efficiently provide services that address the challenges the clients are facing 1 .

Health coaches and psychotherapists both work with the art and science of facilitating change in their patients and clients. While the evolving field of health coaching and the established disciplines of clinical or counseling psychology share major areas of overlap, there are also significant distinctions between the two fields. There is a strong link between behavior and health ${ }^{5}$. Having a healthy lifestyle, e.g., regular physical activity and healthy eating, prevents many diseases. Health coaching can play a role in supporting people to achieve their health goals and changing or maintaining their healthy behavior?

\section{PERSONAL EXPERIENCE:}

As a practitioner in the field of mental health for more than a decade I have witnessed clear distinction in the understanding of the illnesses by the local clients when compared to those from developed nations. The patients from the developed nations would be curious to know or would already know if diagnosed from the service providers or from different resourcesinternet, information brochure, booklets etc. It makes the consultation easy and compliance far better. On the other hand, Nepali natives would not be curious to know or at the most would give up with trivial information. As rightly said, "The First Step to Obtaining Help Is to Accept That Getting Help Is the Right Thing to Do!" the instances of non-compliance is high in our 
practice. In fact, I find it to be one of the most challenging issue. Mostly, our clients leave the consultation room half-heartedly, with lots of questions in mind. Pondering upon this facts I realized that patients or caretakers who knew the disorder were more compliant, could actually monitor the symptoms themselves, and knew the effects of individual medication, the right dose and timing and come for help early. While native clients would usually not take the medicines as advised or stop it once they improve. They would cite examples like; "pharmacist told them not to take", "I continued anti-hypertensive but am alright without psychotropic", self-experimentations are even amazing "I have halved the dose, skipped the medicine for 10 days and nothing happened", in cases of seizure disorders we frequently encounter "my son is taking Phenytoin since last 10 years". "When stopped he develops fainting attacks". "Doctor did not tell us the nature of illness and duration of treatment". "Doctor told us to continue medicine and come on follow up". "As the patient was well we continued treatment". "An experienced acquaintance told us it is not good medicine to take and we stopped" and so on. Same people when asked, "Do you or people you know do the same with other disorders like taking regular antihypertensive, diabetes treatment?" they would bite their tongue, "no. how can we do that". This dichotomy in public's mind is hard to explain. Apart from stigma one very important aspect may be the lack of health coaching. With this in mind from the very beginning in my practice I made sure to coach or give materials to read and follow. There is no denying that we lack time due to pressure of work load and also the back up of psychologist in our regular practice is scarce. Obviously these were not enough as most of them would throw the materials and do not follow instructions. Alternative I chose in last three years of my practice is to empower the nursing staff as a health coach. First, leaflets of major illnesses in Nepali were designed then the nursing staffs were trained to coach. After diagnosis, every clients and or the families are coached about the condition, treatment pros and cons, healthy lifestyle and the role of the family. Over these period we never thought of doing anything out of the box but we were just sure that these are something essential but not done.
It was satisfying to know the world views on these practice through literature. These has added to our practice confidence and evidence. Hope bringing these as an editorial will be justified.

\section{LITERATURE REVIEWS:}

Health coaching appeals to many health care pr ofessionals because it puts the focus squarely on the patient. This is different from the traditional medical model in which health care profesionals who " $k$ now best" define the agenda, terms, and the goals of care. Many clinicians ass ume patients who neglect their health or are non-adherent, "don't see (are in denial or lack insight), don't know, don't know how, or don't care." Based on these assumptions, health care professionals often rely on teaching, instructing, directing and advising patients

to support behavior change. However, for less motivated patients, traditional health educationapproaches are generally ineffe ctive and may lead to negative

clinical outcomes. This is not surprising consider ing that patients are often less concerned with their medical condition(s), than the daily problems that these medical conditions cause or the threats that med ical conditions pose to important life goals or activities.

Various health coaching approaches are being $\mathrm{u}$ sed in employer, primary care,

community, health plan and population health improvement settings to support

individual lifestyle change, treatment adherence, and selfcare. Yet while health

coaching is a frequently used term, it is often a $p$ oorly defined, informal practice.Some clinicians refer to any patient education or advicegiving encounter as "health coaching." Many nonclinician and clinici an health coaches use a variety of life coaching a pproaches that are based more on popular psych ology than behavioral science research. These fa cts raise a number of questions: What is coachin $\mathrm{g}$ ? Can coaching approaches from thebusiness a nd sports worlds be applied by 
clinicians

in health care settings? Which health coaching a pproaches are most effective? How can health coaching be patient centered and deliver best value to patients and health care payers and purchasers ${ }^{6}$ ?

No consensus exists on how to define health coa ching and how to practice healthcoaching. Simil arly, there is little agreement on who should be doing health coaching and who should be receiv ing health coaching. Nor, are there standards for measuring the effectiveness of health coaching e ncounters. Employers may offer healthy employ ees health coaching services to assist in smoking cessation,

healthy diet, weight management or physical act ivity. Primary care providers may offer patients with chronic diseases health coaching to support knowledge, adherence, disease selfcare support, or lifestyle management.

Clinicians in chronic care improvement or diseas e management programs may also offer brief, ph onebased health coaching to support receipt of e videncebased medical care, treatment adherence and daily self-care.

Care managers or case managers, who may not $r$ efer to themselves as "health

coaches," may provide all of the above and more to individuals with multiple or

complex health care conditions.

While a variety of specific health coaching interv entions may be delivered based on the needs of $t$ he population(s) served and the setting(s) in whi ch they are delivered, health coaching services $\mathrm{c}$ an generally be described as either preventive or therapeutic:

\section{PREVENTIVE HEALTH COACHING:}

- Is often referred to as "wellness coaching."

- May be offered by non-clinicians or clinicians.

- Targets individuals with or without chronic co nditions.

-Supports physical activity, healthy diet, or wei ght management as components of general or ta rgeted disease prevention. Is designed to slow $p$ rogression of disease, prevent complications, or support function and independence, e.g., a weig ht- bearing activity program for an individual with osteoporosis.

\section{THERAPEUTIC HEALTH COACHING:}

- Is often referred to as "health coaching."

-Is typically delivered by clinicians, e.g., nurses, physicians, nutritionists.

-Targets individuals affected by one or more chr onic conditions.

-Support physical activity, healthy diet, or weig ht management as components of an evidencebased therapeutic care plan.

-Is designed to slow progression of disease, pre vent complications, or support

function and independence, e.g., a weightbearin g activity program for an

individual with osteoporosis.

What is the recent consensus on Health Coaching?

"A behavioural intervention that facilitates participants in establishing and attaining healthpromoting goals in order to change lifestylerelated behaviours, with the intent of reducing health risks, improving self-management of chronic conditions, and increasing health-related quality of life" 8 . "Instead of treating patients as passive recipients of care, they must be viewed as partners in the business of healing, players in the promotion of health, managers of healthcare resources, and experts on their own circumstances, needs, preferences and capabilities"3. A consensus definition was created in 2013 from 284 research studies which highlights the changing roles of clinicians and patient ${ }^{1}$.

\section{ROLE OF PATIENT:}

A patient-centered approach wherein patients at least partially determine their goals, use selfrecovery and active learning processes together with content education to work towards their goals, and self-monitor behaviours to increase accountability all within the context of an interpersonal relation with a couch.

Role of Clinician

The coach is a healthcare professional trained in behavior change theory, motivational strategies, and communication techniques, which are used to assist patients to develop intrinsic motivation 
and obtain skills to create sustainable change for improved health and wellbeing.

Health coaching is applicable to a broad range of conditions, can be used by all professional groups and is delivered through multiple routes.

Health coaching has been used effectively in smoking cessation, weight reduction, reduction in cardiovascular risk factors, diabetes control, asthma management, readmission management of depression and for medication compliance. Studies suggest that nurses, doctors and allied health professionals may be equally effective as coaches. People with long-term conditions who have received training in health coaching can be just as effective as health professionals. Health coaching can be a standalone intervention, integrated into clinical practice or part of a system of care; carried out by telephone, on line, face to face or in groups ${ }^{1}$.

\section{DISCUSSION:}

As clinicians we are not abandoning our traditional medical skills of assessment, diagnosis and treatment. However, the challenge for us is to look beyond clinical recovery and to measure effectiveness of treatments and interventions in terms of the impact of these on the goals and outcomes that matter to the individual service user and their family. We need to continually ask ourselves are we helping or hindering a person in their recovery ${ }^{2}$. Health coaching is a partnership and different type of conversation between clinicians and patients that guides and prompts patients to be more active participants in their care and behavior change.

The evidence is that there are many benefits associated with health coaching. In order for it to be fully effective health coaching may need to be implemented as part of a wider programme supporting education and behavior change ${ }^{1}$. Hope this is will open a new prospect for fulfilling experience to the practitioners and extra beneficial service to the communities.

\section{REFERENCES:}

1. Better conversation. A guide to health coaching. The health coaching coalition. URL: www.betterconversation.co.uk.

2. Boardman J, Craig T, Goddard C, Henderson C, McCarthy J, Mclnerny T, Cohen A, Potter M, Rinaidi $M$ and Whicher E. Recovery is for All. Hope, Agency and Opportunity in Psychiatry. A position statement by consultant psychiatrists. South London and Mauldsley NHS Foundation Trust and South West London and St George's Mental Health NHS Trust (2010). London: SLAM/SWLSTG.

3. Coulter A. The health coaching approach: $A$ different way to talk with patients. (C) NHS Institute for Innovation and Improvement, 2013

4. Hart V, Blattner $J$ and Leipsic S. Coaching versus Therapy: A perspective. Consulting Psychology Journal: Practice and Research, Vol.53, No.4, 229-237.

5. Institute of Medicine: Health and Behavior: The Interplay of Biological, Behavioral, and Societal Influences. National Academies Press, Washington (2001).

6. Moving to an EvidenceBased Health Coaching Practice. Retrieved from: http://infocus.healthsciences.org/HSI_Articles.ht $\underline{m l}$.

7. R. Orji, M. Reisinger, M. Busch, A. Dijkstra, A. Stibe, M. Tscheligi (eds.): Understanding Effective Coaching on Healthy Lifestyle by Combining Theory- and Data-driven Approaches. In: Proceedings of the Personalization in Persuasive Technology Workshop, Persuasive Technology 2016, Salzburg, Austria, 05-04-2016, published at http://ceur-ws.org.

8. Ryn $V$ and Heaney. The health coaching approach: A different way to talk with patients. (C) NHS Institute for Innovation and Improvement, 2013. 